\title{
钉催化芳香硝基化合物和脂肪三级胺反应一锅法合成喹啉衍生物
}

\author{
杨辉琼*, $a, b$ 谌 儒 $b$ 邓国军 $b$ \\ ( ${ }^{a}$ 湖南工程学院化学化工学院 湘潭 411104)

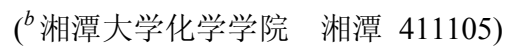

\begin{abstract}
摘要 以芳香硝基化合物和脂肪三级胺为原料, 三环已基膦四氟硼酸盐为配体, 在三氯化钓催化下 “一锅法” 合成了 一系列喹啉衍生物. 该法不需要添加任何额外的还原剂, 原料便宜易得, 操作工艺简单, 产率中等. 产物结构通过 IR, ${ }^{1} \mathrm{H} N M R,{ }^{13} \mathrm{C}$ NMR, MS 以及元素分析表征.

关键词 $\mathrm{C}-\mathrm{N}$ 键形成; 一锅法; 喹啉衍生物; 钉催化; 硝基芳烃; 三级胺
\end{abstract}

\section{Ruthenium-Catalyzed One-Pot Synthesis of Quinolines from Nitroarenes and Trialkylamines}

\author{
Yang, Huiqiong* ${ }^{*, a, b} \quad{\text { Chen, } \mathrm{Ru}^{b} \quad \text { Deng, Guojun }}^{b}$ \\ $\left({ }^{a}\right.$ College of Chemistry and Chemical Engineering, Hunan Institute of Engineering, Xiangtan 411104) \\ $\left({ }^{b}\right.$ College of Chemistry, Xiangtan University, Xiangtan 411105)
}

\begin{abstract}
A series of quinolines were synthesized in moderate yields by one-pot reaction of nitroarenes and trialkylamines in the presence of a catalytic amount of ruthenium trichloride catalyst together with tricyclohexylphosphonium tetrafluoroborate ligand. This method is convenient without extra reductant, and nitroarenes are cheap and highly available. The structures of the products were characterized by IR, ${ }^{1} \mathrm{H}$ NMR, ${ }^{13} \mathrm{C}$ NMR, MS techniques and elemental analysis.

Keywords $\quad \mathrm{C}-\mathrm{N}$ bond formation; one-pot synthesis; quinolines; ruthenium catalyst; nitroarenes; trialkylamines
\end{abstract}

喹啉及其衍生物是一类十分重要的氮杂环结构的 化合物，广泛用于合成医药、染料、农药、食品饲料添 加剂、抗氧化剂、缓蚀剂、感光材料、溶剂和萃取剂等. 特别是在医药工业中, 喹啉类衍生物大多具有抗症

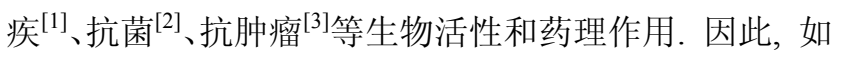
何用化学方法来合成喹啉类衍生物一直是研究的热点. 到目前为止, 已发展出的合成喹啉类衍生物的方法有 Skraup 法 ${ }^{[4]} 、$ Doebner-Vonmiller 法 ${ }^{[5]} 、$ Cambes 法 ${ }^{[6]}$ 、 Conrad-Limpach 法 ${ }^{[7]}$ 、Friedlander 法 $^{[8]}$ 以及 Camps 法 ${ }^{[0]}$ 等, 但它们大都以芳胺为原料, 经多步反应来合成, 存 在原料芳胺易氧化、操作步骤长、分离麻烦等缺点. 近 年来, “一锅法” ${ }^{[10]}$ 合成哇啉类化合物的研究也得到了 广泛关注. 相比而言, “一锅法” 具有以下特点: (1)采用 简单的原料来合成结构复杂的目标产物; (2)成效率高, 一步可以形成多个化学键; (3) 只进行一次后处理过程, 不仅节省了人力, 而且大大减少了后处理过程中各种试
剂的使用.

现今, 过渡金属催化备受有机化学工作者关注. 有 文献[11]报道过渡金属催化胺交换反应可以合成 $\mathrm{N}$-杂 环化合物和不对称胺, 它主要通过烷基胺中的烷基转移 来实现. 近来, 我们课题组在以芳香硝基化合物和醇为 反应物用于构建 $\mathrm{C}-\mathrm{N}$ 键的研究 ${ }^{[12]}$ 中发现, 硝基可以利 用醇氧化为醛的过程中释放的氢实现原位还原, 进而选 择性生成二级胺、三级胺和酰胺. 在这一过程中多次利 用 “借氢战略”, 整个反应过程不需要添加额外的还原 剂和氧化剂, 实现了醇氧化、硝基还原、亚胺生成、亚 胺还原多步反应一锅完成. 为了进一步研发“借氢战略” 在 $\mathrm{C}-\mathrm{N}$ 键生成反应中的应用, 我们研究了芳香硝基化 合物和脂肪三级胺的反应, 利用胺 $\mathrm{N}$ 邻位的亚甲基易被 活化生成亚胺过程中释放出来的氢实现硝基的原位还 原, 进而合成喹啉及其衍生物. 这克服了以前合成方法 的缺点, 该法不需要添加任何额外的还原剂, 原料芳香

\footnotetext{
* E-mail: yanghuiqiong2996@126.com

Received February 23, 2012; revised May 16, 2012; published online June 1, 2012.

Project supported by the National Natural Science Foundation of China (No. 20902076).

国家自然科学基金(No. 20902076)资助项目.
} 
硝基化合物稳定、便宜、易得，操作工艺简单.

本文以芳香硝基化合物和脂肪三级胺为底物, 在钌 化合物的催化下“一锅法”合成了一系列喹啉衍生物. 并 对目标产物进行了 IR, ${ }^{1} \mathrm{H} N M R,{ }^{13} \mathrm{C} N \mathrm{NR}, \mathrm{MS}$ 以及元素 分析等表征.

\section{1 实验部分}

\section{1 仪器与试剂}

熔点由 WRS-1B 型数字熔点仪测定(温度计未校 正); 红外光谱采用 Nicolet Avatar-370 型傅里叶红外光 谱仪测定; 核磁共振氢谱和碳谱采用 Bruker-AV 型 (400 和 $100 \mathrm{MHz}$ ) 核磁共振仪测定, 以 $\mathrm{CDCl}_{3}$ 为溶剂、 TMS 为内标; 质谱采用 Agilent 5975 GC-MS 气质联用 仪测定; 元素分析采用 PE-2400 型元素分析仪测定.

所用试剂和溶剂均为市售分析纯. 钉盐从 AlfaAesar 公司购买.

\section{2 喹啉衍生物的合成}

在 $10 \mathrm{~mL}$ 干燥的微波反应管中依次加入 $\mathrm{RuCl}_{3}$ ・ $3 \mathrm{H}_{2} \mathrm{O}$ (0.02 mmol), $\mathrm{PCy}_{3} \mathrm{HBF}_{4}$ (0.04 mmol), 芳香硝基化 合物 $(1,0.6 \mathrm{mmol})$ 后, 抽真空并用氩气置换三次, 再用 注射器向密封的反应管中加入脂肪三级胺 $(2,0.2 \mathrm{mmol})$ 和甲苯 $(0.2 \mathrm{~mL})$, 将反应管置于 $160{ }^{\circ} \mathrm{C}$ 的油浴中反应 $24 \mathrm{~h}$ 后取出, 冷却至室温, 低沸点物质通过真空抽除, 残液通过柱层析分离 [硅胶柱, $V$ (石油醚) : $V($ 乙 酸乙 酯) $=98 ： 2$ ], 得到纯的喹啉衍生物 $\mathbf{3}$.

3-乙基-6-甲基-2-丙基喹啉(3a) ${ }^{[13]}$ : 产率 $62 \%$, 黄色 油状液体. ${ }^{1} \mathrm{H}$ NMR (400 MHz, $\left.\mathrm{CDCl}_{3}\right) \delta: 7.92(\mathrm{~d}, J=8.5$ $\mathrm{Hz}, 1 \mathrm{H}), 7.79$ (s, 1H), 7.51 (s, 1H), 7.46 (d, $J=8.5 \mathrm{~Hz}$, $1 \mathrm{H}), 2.96$ (t, $J=8.0 \mathrm{~Hz}, 2 \mathrm{H}), 2.84$ (q, $J=7.4 \mathrm{~Hz}, 2 \mathrm{H}), 2.52$ (s, 3H), 1.84 (sextet, $J=6.1 \mathrm{~Hz}, 2 \mathrm{H}), 1.35$ (t, $J=7.4 \mathrm{~Hz}$, $3 \mathrm{H}), 1.08$ (t, $J=7.3 \mathrm{~Hz}, 3 \mathrm{H}) ;{ }^{13} \mathrm{C} \mathrm{NMR}\left(100 \mathrm{MHz}, \mathrm{CDCl}_{3}\right.$ ) $\delta: 160.9,145.1,135.3,135.2,133.3,130.5,128.2,127.4$, 125.8, 37.7, 25.2, 22.8, 21.4, 14.4, 14.2; MS (EI) $m / z(\%)$ : 213, 198, 185 (100), 157, 128.

3-乙基-2-丙基喹啉(3b) ${ }^{[13]}$ : 产率 $68 \%$, 亮黄色油状 液体. ${ }^{1} \mathrm{H}$ NMR $\left(400 \mathrm{MHz}, \mathrm{CDCl}_{3}\right) \delta: 8.04(\mathrm{~d}, J=8.5 \mathrm{~Hz}$, 1H), 7.89 (s, 1H), 7.75 (d, $J=8.0 \mathrm{~Hz}, 1 \mathrm{H}), 7.63$ (t, $J=7.2$ $\mathrm{Hz}, 1 \mathrm{H}), 7.47$ (t, $J=7.4 \mathrm{~Hz}, 1 \mathrm{H}), 2.95$ (t, $J=7.9 \mathrm{~Hz}, 2 \mathrm{H})$, 2.87 (q, $J=7.4 \mathrm{~Hz}, 2 \mathrm{H}$ ), 1.85 (sextet, $J=7.6 \mathrm{~Hz}, 2 \mathrm{H}$ ), 1.37 $(\mathrm{t}, J=7.5 \mathrm{~Hz}, 3 \mathrm{H}), 1.09(\mathrm{t}, J=7.3 \mathrm{~Hz}, 3 \mathrm{H}) ;{ }^{13} \mathrm{C}$ NMR $(100$ $\left.\mathrm{MHz}, \mathrm{CDCl}_{3}\right) \delta: 161.9,146.5,135.4,133.8,128.5,127.4$, $126.8,125.5,14.0,37.7,25.2,22.7,14.4,14.3$; MS (EI) $\mathrm{m} / z(\%): 199,184,171$ (100), 143, 128.

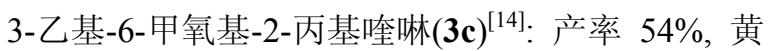
色油状液体. ${ }^{1} \mathrm{H}$ NMR (400 $\left.\mathrm{MHz} \mathrm{CDCl}_{3}\right) \delta: 7.92(\mathrm{~d}, J=$
$9.1 \mathrm{~Hz}, 1 \mathrm{H}), 7.79$ (s, 1H), 7.29 (d, $J=5.7 \mathrm{~Hz}, 1 \mathrm{H}), 7.04$ (s, 1H), 3.9 (s, 3H), 2.94 (t, $J=7.9 \mathrm{~Hz}, 2 \mathrm{H}), 2.84$ (q, $J=7.4$ $\mathrm{Hz}, 2 \mathrm{H}$ ), 1.84 (sextet, $J=7.5 \mathrm{~Hz}, 2 \mathrm{H}), 1.35$ (t, $J=7.4 \mathrm{~Hz}$, $3 \mathrm{H}), 1.07$ (t, $J=7.3 \mathrm{~Hz}, 3 \mathrm{H}) ;{ }^{13} \mathrm{C} \mathrm{NMR}\left(100 \mathrm{MHz}, \mathrm{CDCl}_{3}\right)$ $\delta: 159.3,157.2,142.539,135.6,132.9,129.9,128.2,120.7$, $104.8,55.4,37.5,25.2,22.8,14.4,14.2 ; \mathrm{MS}(\mathrm{EI}) \mathrm{m} / z(\%)$ : 229, 214, 201 (100), 173, 115.

6-氯-3-乙基-2-丙基喹啉(3d) ${ }^{[13]}$ ：产率 $26 \%$ ，黄色油 状液体. ${ }^{1} \mathrm{H}$ NMR $\left(400 \mathrm{MHz}, \mathrm{CDCl}_{3}\right) \delta: 7.95$ (d, $J=8.9$ $\mathrm{Hz}, 1 \mathrm{H}), 7.79$ (s, 1H), 7.73 (s, 1H), 7.56 (d, $J=9.0 \mathrm{~Hz}$, 1H), 2.96 (t, $J=7.9 \mathrm{~Hz}, 2 \mathrm{H}), 2.85$ (q, $J=7.4 \mathrm{~Hz}, 2 \mathrm{H}), 1.85$ (sextet, $J=7.5 \mathrm{~Hz}, 2 \mathrm{H}), 1.36(\mathrm{t}, J=7.5 \mathrm{~Hz}, 3 \mathrm{H}), 1.08$ (t, $J=7.3 \mathrm{~Hz}, 3 \mathrm{H}) ;{ }^{13} \mathrm{C} \mathrm{NMR}\left(100 \mathrm{MHz}, \mathrm{CDCl}_{3}\right) \delta: 162.3$, $144.8,136.4,132.8,131.1,130.2,129.1,127.9,125.5$, 37.6, 29.6, 25.1, 22.5, 14.2; MS (EI) $m / z$ (\%): 233, 218, 205 (100), 177, 127.

6-溴-3-乙基-2-丙基喹啉 $(3 \mathbf{e})$ ：产率 32\%，黄色油状 液体. ${ }^{1} \mathrm{H}$ NMR $\left(400 \mathrm{MHz}, \mathrm{CDCl}_{3}\right) \delta: 7.90(\mathrm{~s}, 1 \mathrm{H}), 7.81(\mathrm{~d}$, $J=9.3 \mathrm{~Hz}, 1 \mathrm{H}), 7.78$ (s, 4H), 7.69 (d, $J=9.2 \mathrm{~Hz}, 1 \mathrm{H}), 2.97$ (t, $J=7.9 \mathrm{~Hz}, 2 \mathrm{H}), 2.85$ (q, $J=7.4 \mathrm{~Hz}, 2 \mathrm{H}$ ), 1.85 (sextet, $J=7.5 \mathrm{~Hz}, 2 \mathrm{H}), 1.35(\mathrm{t}, J=7.5 \mathrm{~Hz}, 3 \mathrm{H}), 1.08(\mathrm{t}, J=7.3$ $\mathrm{Hz}, 3 \mathrm{H}) ;{ }^{13} \mathrm{C} \mathrm{NMR}\left(100 \mathrm{MHz}, \mathrm{CDCl}_{3}\right) \delta: 162.5,145.0$, 136.4, 132.7, 131.6, 130.3, 128.9, 128.5, 119.2, 37.7, 25.1, 22.5, 14.2, 14.2; IR (neat) $v: 3062,2963,2932,2872$, 1594, 1556, 1476, 915, 828, $559 \mathrm{~cm}^{-1}$; MS (EI) $\mathrm{m} / \mathrm{z}(\%)$ : 277, 262, 249 (100), 167, 115. Anal. calcd for $\mathrm{C}_{14} \mathrm{H}_{16} \mathrm{NBr}$ : C 60.44, H 5.80, N 5.03; found C 60.56, H 5.61, N 5.13.

3-乙基-7-甲基-2-丙基喹啉(3f ${ }^{[13]}$ : 产率 75\%, 黄色 油状液体. ${ }^{1} \mathrm{H}$ NMR $\left(400 \mathrm{MHz}, \mathrm{CDCl}_{3}\right) \delta: 8.03(\mathrm{~s}, 1 \mathrm{H})$, $7.83(\mathrm{~s}, 1 \mathrm{H}), 7.64(\mathrm{~d}, J=8.2 \mathrm{~Hz}, 1 \mathrm{H}), 7.30$ (d, $J=8.7 \mathrm{~Hz}$, 1H), 2.96 (t, $J=8.0 \mathrm{~Hz}, 2 \mathrm{H}), 2.85$ (q, $J=7.4 \mathrm{~Hz}, 2 \mathrm{H}$ ), 2.55 (s, 3H), 1.84 (sextet, $J=7.5 \mathrm{~Hz}, 2 \mathrm{H}), 1.35$ (t, $J=8.0 \mathrm{~Hz}$, $3 \mathrm{H}), 1.08$ (t, $J=7.3 \mathrm{~Hz}, 3 \mathrm{H}) ;{ }^{13} \mathrm{C} \mathrm{NMR}\left(100 \mathrm{MHz}, \mathrm{CDCl}_{3}\right)$ $\delta: 161.8,146.7,138.3,134.5,133.6,127.7,127.6,126.6$, 125.4, 37.8, 25.2, 22.8, 21.8, 14.5, 14.3; MS (EI) $m / z(\%)$ : 213, 198, 185 (100), 157, 115.

3-乙基-7-碘-2-丙基喹啉(3g)：产率 63\%，黄色油状 液体. ${ }^{1} \mathrm{H}$ NMR (400 MHz, $\left.\mathrm{CDCl}_{3}\right) \delta: 8.47(\mathrm{~s}, 1 \mathrm{H}), 7.83(\mathrm{~s}$, 2H), $7.71(\mathrm{~d}, J=8.4 \mathrm{~Hz}, 1 \mathrm{H}), 7.46$ (d, $J=8.5 \mathrm{~Hz}, 1 \mathrm{H}), 2.95$ (t, $J=7.8 \mathrm{~Hz}, 2 \mathrm{H}), 2.83$ (q, $J=7.4 \mathrm{~Hz}, 2 \mathrm{H}$ ), 1.85 (sextet, $J=7.5 \mathrm{~Hz}, 2 \mathrm{H}), 1.35$ (t, $J=7.5 \mathrm{~Hz}, 3 \mathrm{H}), 1.08$ (t, $J=7.3$ $\mathrm{Hz}, 3 \mathrm{H}) ;{ }^{13} \mathrm{C} \mathrm{NMR}\left(100 \mathrm{MHz}, \mathrm{CDCl}_{3}\right) \delta: 162.8,147.3$, $137.7,136.1,134.2,133.6,128.1,126.3,93.8,37.6,25.2$, 22.4, 14.3, 14.2; IR (neat) $v: 3056,2962,2930,2871$, $1589,1551,1474,917,854,476 \mathrm{~cm}^{-1}$; MS (EI) $\mathrm{m} / z(\%)$ : 
325, 310, 297 (100), 167, 127. Anal. calcd for $\mathrm{C}_{14} \mathrm{H}_{16} \mathrm{NI}$ : C 51.71, H 4.96, N 4.31; found C 51.89, H 4.78, N 4.45.

3-乙基-8-甲基-2-丙基喹啉(3h) ${ }^{[13]}$ : MS (EI) $m / z$ (\%): 213, 198, 185 (100), 157, 128.

3-乙基-6,8-二甲基-2-丙基喹啉(3i) ${ }^{[15]}$ : 产率 40\%, 黄色油状液体. ${ }^{1} \mathrm{H}$ NMR $\left(400 \mathrm{MHz}, \mathrm{CDCl}_{3}\right) \delta: 7.71(\mathrm{~s}$, 1H), 7.32 (s, 1H), 7.29 (s, 1H), 2.93 (t, $J=7.6 \mathrm{~Hz}, 2 \mathrm{H}$ ), 2.80 (q, $J=7.5 \mathrm{~Hz}, 2 \mathrm{H}), 2.75$ (s, 3H), 2.45 (s, 3H), 1.92 (sextet, $J=7.4 \mathrm{~Hz}, 2 \mathrm{H}), 1.31$ (t, $J=7.5 \mathrm{~Hz}, 3 \mathrm{H}), 1.06$ (t, $J=7.4 \mathrm{~Hz}, 3 \mathrm{H}) ;{ }^{13} \mathrm{C}$ NMR $\left(100 \mathrm{MHz}, \mathrm{CDCl}_{3}\right) \delta: 159.2$, $144.1,136.3,135.0,134.6,133.1,130.5,127.2,123.7$, 37.3, 25.1, 21.8, 21.5, 17.6, 14.4, 14.2; MS (EI) $\mathrm{m} / z$ (\%): 227, 212, 199 (100), 171, 115.

3-乙基-2-丙基苯并喹啉(3j $)^{[16]}$ : 产率 82\%, 亮黄色 油状液体. ${ }^{1} \mathrm{H}$ NMR $\left(400 \mathrm{MHz}, \mathrm{CDCl}_{3}\right) \delta: 9.35(\mathrm{~d}, J=7.4$ $\mathrm{Hz}, 1 \mathrm{H}), 7.90$ (s, 1H), 7.89 (d, $J=6.6 \mathrm{~Hz}, 1 \mathrm{H}), 7.64 \sim 7.64$ $(\mathrm{m}, 4 \mathrm{H}), 3.06(\mathrm{t}, J=7.6 \mathrm{~Hz}, 2 \mathrm{H}), 2.89$ (q, $J=7.5 \mathrm{~Hz}, 2 \mathrm{H})$, 2.05 (sextet, $J=7.5 \mathrm{~Hz}, 2 \mathrm{H}$ ), 1.38 (t, $J=7.5 \mathrm{~Hz}, 3 \mathrm{H}$ ), 1.14 (t, $J=7.3 \mathrm{~Hz}, 3 \mathrm{H}) ;{ }^{13} \mathrm{C}$ NMR $\left(100 \mathrm{MHz}, \mathrm{CDCl}_{3}\right) \delta: 159.8$, $144.0,135.8,133.9,131.7,127.6,127.3,126.6,126.5$, 125.1, 124.8, 124.4, 37.3, 25.2, 22.1, 14.5, 14.3; MS (EI) $\mathrm{m} / \mathrm{z}$ (\%): 249, 234, 221 (100), 193, 109.

7-乙基-6-丙基-4,5-噻唑喹啉(3k): 产率 62\%, 乳白 色固体. m.p. 105.9 107.2 ${ }^{\circ} \mathrm{C} ;{ }^{1} \mathrm{H}$ NMR (400 MHz, $\left.\mathrm{CDCl}_{3}\right) \delta: 9.30(\mathrm{~s}, 1 \mathrm{H}), 9.21(\mathrm{~s}, 1 \mathrm{H}), 8.67(\mathrm{~s}, 1 \mathrm{H}), 8.60$ (s, $1 \mathrm{H}), 3.57$ (t, $J=7.2 \mathrm{~Hz}, 2 \mathrm{H}), 3.06$ (q, $J=7.1 \mathrm{~Hz}, 2 \mathrm{H})$, $1.99 \sim 2.06(\mathrm{~m}, 2 \mathrm{H}), 1.51(\mathrm{t}, J=7.0 \mathrm{~Hz}, 3 \mathrm{H}), 1.20(\mathrm{t}, J=$ $6.9 \mathrm{~Hz}, 3 \mathrm{H}) ;{ }^{13} \mathrm{C}$ NMR (100 MHz, $\left.\mathrm{CDCl}_{3}\right) \delta: 159.6,155.2$, 153.1, 139.4, 138.1, 130.4, 129.3, 123.3, 120.7, 33.2, 24.1, 22.6, 14.6, 14.2; IR (KBr) v: 3084, 2963, 2930, 2873, 1636, 1558, 1457, 1406, 1282, 1125, 1089, 1020, 856, 685, $525 \mathrm{~cm}^{-1}$; MS (EI) $\mathrm{m} / \mathrm{z}$ (\%): 256, 241, 228 (100), 213, 200, 114. Anal. calcd for $\mathrm{C}_{15} \mathrm{H}_{16} \mathrm{~N}_{2} \mathrm{~S}$ : C 70.27, H 6.29, N 10.93; found C 70.39, H 6.04, N 11.05 .

2-乙基-3,6-二甲基喹啉(31 ${ }^{[17]}$ : 产率 52\%, 亮黄色油 状液体. ${ }^{1} \mathrm{H}$ NMR $\left(400 \mathrm{MHz}, \mathrm{CDCl}_{3}\right) \delta: 7.92(\mathrm{~d}, J=8.4$ $\mathrm{Hz}, 1 \mathrm{H}), 7.77$ (s, 1H), 7.48 (s, 1H), $7.46(\mathrm{~d}, J=10.6 \mathrm{~Hz}$, $1 \mathrm{H}), 2.99$ (q, $J=7.5 \mathrm{~Hz}, 2 \mathrm{H}), 2.52(\mathrm{~s}, 3 \mathrm{H}), 2.49(\mathrm{~s}, 3 \mathrm{H})$, 1.38 (t, $J=7.5 \mathrm{~Hz}, 3 \mathrm{H}) ;{ }^{13} \mathrm{C}$ NMR $\left(100 \mathrm{MHz}, \mathrm{CDCl}_{3}\right) \delta$ : $162.2,145.3,135.1,130.4,129.2,128.3,127.4,125.5$, 29.3, 21.4, 19.0, 12.7; MS (EI) $m / z$ (\%): 184 (100), 157, 128,90 .

3-丁基-6-甲基-2-戊基哇啉(3m) ${ }^{[18]}$ : 产率 74\%, 黄色 油状液体. ${ }^{1} \mathrm{H}$ NMR $\left(400 \mathrm{MHz}, \mathrm{CDCl}_{3}\right) \delta: 7.91$ (d, $J=8.5$ $\mathrm{Hz}, 1 \mathrm{H}), 7.76$ (s, 1H), 7.49 (s, 1H), 7.45 (d, $J=8.5 \mathrm{~Hz}$,
1H), 2.96 (t, $J=8.1 \mathrm{~Hz}, 2 \mathrm{H}), 2.78$ (t, $J=7.8 \mathrm{~Hz}, 2 \mathrm{H}), 2.52$ (s, $3 \mathrm{H}), 1.78 \sim 1.82(\mathrm{~m}, 2 \mathrm{H}), 1.64 \sim 1.70(\mathrm{~m}, 4 \mathrm{H}), 1.40 \sim$ 1.50 (m, 4H), 1.00 (t, $J=7.3 \mathrm{~Hz}, 3 \mathrm{H}), 0.93$ (t, $J=7.0 \mathrm{~Hz}$, $3 \mathrm{H}) ;{ }^{13} \mathrm{C}$ NMR $\left(100 \mathrm{MHz}, \mathrm{CDCl}_{3}\right) \delta: 161.2,145.1,135.1$, 134.1, 134.0, 130.5, 128.2, 127.2, 125.7, 35.8, 32.7, 32.1, 32.1, 29.4, 22.6, 22.6, 21.4, 14.0, 13.9; MS (EI) $m / z$ (\%): 240, 226, 198, $171(100)$.

2-庚基-3-已基-6-甲基喹啉 $(3 n)^{[15]}$ : 产率 75\%，黄色 油状液体. ${ }^{1} \mathrm{H}$ NMR $\left(400 \mathrm{MHz}, \mathrm{CDCl}_{3}\right) \delta: 7.91$ (d, $J=8.5$ Hz, 1H), 7.76 (s, 1H), 7.49 (s, 1H), 7.45 (d, $J=8.6 \mathrm{~Hz}$, $1 \mathrm{H}), 2.96(\mathrm{t}, J=8.0 \mathrm{~Hz}, 2 \mathrm{H}), 2.78(\mathrm{t}, J=7.8 \mathrm{~Hz}, 2 \mathrm{H}), 2.52$ $(\mathrm{s}, 3 \mathrm{H}), 1.77 \sim 1.81(\mathrm{~m}, 2 \mathrm{H}), 1.65 \sim 1.71(\mathrm{~m}, 2 \mathrm{H}), 1.31 \sim$ $1.47(\mathrm{~m}, 14 \mathrm{H}), 0.89 \sim 0.94(\mathrm{~m}, 6 \mathrm{H}) ;{ }^{13} \mathrm{C} \mathrm{NMR}(100 \mathrm{MHz}$, $\left.\mathrm{CDCl}_{3}\right) \delta: 161.3,145.1,135.1,134.1,134.0,130.5,128.2$, 127.2, 125.7, 35.8, 32.4, 31.8, 31.7, 30.5, 29.9, 29.7, 29.2, 29.2, 22.6, 22.5, 21.4, 14.0; MS (EI) $\mathrm{m} / z$ (\%): 268, 226, 184, 171 (100).

3-乙基-6-甲基-2-丙基喹啉(3o ${ }^{[13]}$ : 产率 35\%, 黄色 油状液体. ${ }^{1} \mathrm{H}$ NMR $\left(400 \mathrm{MHz}, \mathrm{CDCl}_{3}\right) \delta: 7.92(\mathrm{~d}, J=8.5$ $\mathrm{Hz}, 1 \mathrm{H}), 7.79$ (s, 1H), 7.51 (s, 1H), 7.46 (d, $J=8.5 \mathrm{~Hz}$, $1 \mathrm{H}), 2.96$ (t, $J=8.0 \mathrm{~Hz}, 2 \mathrm{H}), 2.84$ (q, $J=7.4 \mathrm{~Hz}, 2 \mathrm{H}), 2.52$ (s, 3H), 1.84 (sextet, $J=6.1 \mathrm{~Hz}, 2 \mathrm{H}), 1.35$ (t, $J=7.4 \mathrm{~Hz}$, $3 \mathrm{H}), 1.08$ (t, $J=7.3 \mathrm{~Hz}, 3 \mathrm{H}) ;{ }^{13} \mathrm{C}$ NMR $\left(100 \mathrm{MHz}, \mathrm{CDCl}_{3}\right)$ $\delta: 160.9,145.1,135.3,135.2,133.3,130.5,128.2,127.4$, $125.8,37.7,25.2,22.8,21.4,14.4,14.2$; MS (EI) $\mathrm{m} / z(\%)$ : 213, 198, 185 (100), 157, 115.

\section{2 结果与讨论}

\section{1 反应条件的优化}

我们首先选用对硝基甲苯(1a)与三丁胺 (2a)为反应 模板, 进行催化剂、配体和溶剂等条件优化, 结果见表 1 .

由表 1 中 Entry 1 数据可以看出, 当选用 $\mathrm{RuCl}_{3}$ ・ $3 \mathrm{H}_{2} \mathrm{O}$ 为催化剂, 不加任何配体, 甲苯为溶剂时, 通过 GC-MS 能检测到有 $20 \%$ 的产物生成. 在相同的条件下 我们对不同的配体进行了考察(表 1, Entries 2 8), 当选 用膦配体时，部分膦配体能够较好地对金属钓进行活 化, 从而促进反应的进行. 使我们高兴的是，当用 $\mathrm{PCy}_{3} \mathrm{HBF}_{4}$ 为配体时, 目标产物的产率达到 $84 \%$; 但是 在选用氮配体时发现不能促进反应的发生, 通过 GC-MS 只能检测到微量的产物生成, 可能在这个反应 体系中氮配体不能对金属钓进行活化. 然后，在选定 $\mathrm{PCy}_{3} \mathrm{HBF}_{4}$ 为配体时, 研究其它钉盐的催化性能(表 1, Entries 9 12), 发现还是 $\mathrm{RuCl}_{3} \cdot 3 \mathrm{H}_{2} \mathrm{O}$ 的催化效果最好; 再选定 $\mathrm{RuCl}_{3} \cdot 3 \mathrm{H}_{2} \mathrm{O}$ 为催化剂、 $\mathrm{PCy}_{3} \mathrm{HBF}_{4}$ 为配体, 研究 
表 1 反应条件的优化 ${ }^{a}$

Table 1 Optimization of reaction conditions

\begin{tabular}{|c|c|c|c|c|}
\hline Entry & Catalyst & Ligand & Solvent & Yield ${ }^{b} / \%$ \\
\hline 1 & $\mathrm{RuCl}_{3} \cdot 3 \mathrm{H}_{2} \mathrm{O}$ & None & Toluene & 20 \\
\hline 2 & $\mathrm{RuCl}_{3} \cdot 3 \mathrm{H}_{2} \mathrm{O}$ & dppb & Toluene & 40 \\
\hline 3 & $\mathrm{RuCl}_{3} \cdot 3 \mathrm{H}_{2} \mathrm{O}$ & $\mathrm{PPh}_{3}$ & Toluene & 29 \\
\hline 4 & $\mathrm{RuCl}_{3} \cdot 3 \mathrm{H}_{2} \mathrm{O}$ & dppe & Toluene & 62 \\
\hline 5 & $\mathrm{RuCl}_{3} \cdot 3 \mathrm{H}_{2} \mathrm{O}$ & $\mathrm{PCy}_{3} \mathrm{HBF}_{4}$ & Toluene & 84 \\
\hline 6 & $\mathrm{RuCl}_{3} \cdot 3 \mathrm{H}_{2} \mathrm{O}$ & BINAP & Toluene & 15 \\
\hline 7 & $\mathrm{RuCl}_{3} \cdot 3 \mathrm{H}_{2} \mathrm{O}$ & 1,10-Phen & Toluene & Trance \\
\hline 8 & $\mathrm{RuCl}_{3} \cdot 3 \mathrm{H}_{2} \mathrm{O}$ & DMAP & Toluene & Trance \\
\hline 9 & {$\left[\mathrm{Ru}(p \text {-cymene }) \mathrm{Cl}_{2}\right]_{2}$} & $\mathrm{PCy}_{3} \mathrm{HBF}_{4}$ & Toluene & 50 \\
\hline 10 & {$\left[\mathrm{RuCl}_{2}(\mathrm{COD})\right]_{n}$} & $\mathrm{PCy}_{3} \mathrm{HBF}_{4}$ & Toluene & 53 \\
\hline 11 & $\mathrm{Ru}(\mathrm{acac})_{3}$ & $\mathrm{PCy}_{3} \mathrm{HBF}_{4}$ & Toluene & 17 \\
\hline 12 & $\mathrm{Ru}(\mathrm{CO})(\mathrm{H})_{2}\left(\mathrm{PPh}_{3}\right)_{3}$ & $\mathrm{PCy}_{3} \mathrm{HBF}_{4}$ & Toluene & 13 \\
\hline 13 & $\mathrm{RuCl}_{3} \cdot 3 \mathrm{H}_{2} \mathrm{O}$ & $\mathrm{PCy}_{3} \mathrm{HBF}_{4}$ & Xylene & 55 \\
\hline 14 & $\mathrm{RuCl}_{3} \cdot 3 \mathrm{H}_{2} \mathrm{O}$ & $\mathrm{PCy}_{3} \mathrm{HBF}_{4}$ & DMF & 38 \\
\hline 15 & $\mathrm{RuCl}_{3} \cdot 3 \mathrm{H}_{2} \mathrm{O}$ & $\mathrm{PCy}_{3} \mathrm{HBF}_{4}$ & DMSO & 0 \\
\hline 16 & $\mathrm{RuCl}_{3} \cdot 3 \mathrm{H}_{2} \mathrm{O}$ & $\mathrm{PCy}_{3} \mathrm{HBF}_{4}$ & NMP & 14 \\
\hline 17 & $\mathrm{RuCl}_{3} \cdot 3 \mathrm{H}_{2} \mathrm{O}$ & $\mathrm{PCy}_{3} \mathrm{HBF}_{4}$ & None & 72 \\
\hline $18^{c}$ & $\mathrm{RuCl}_{3} \cdot 3 \mathrm{H}_{2} \mathrm{O}$ & $\mathrm{PCy}_{3} \mathrm{HBF}_{4}$ & Toluene & 60 \\
\hline $19^{d}$ & $\mathrm{RuCl}_{3} \cdot 3 \mathrm{H}_{2} \mathrm{O}$ & $\mathrm{PCy}_{3} \mathrm{HBF}_{4}$ & Toluene & 64 \\
\hline $20^{e}$ & $\mathrm{RuCl}_{3} \cdot 3 \mathrm{H}_{2} \mathrm{O}$ & $\mathrm{PCy}_{3} \mathrm{HBF}_{4}$ & Toluene & 49 \\
\hline
\end{tabular}

${ }^{a}$ Conditions: 1a $(0.6 \mathrm{mmol}), \mathbf{2 a}(0.2 \mathrm{mmol})$, catalyst $(0.02 \mathrm{mmol})$, ligand $(0.04$ mmol), solvent $(0.2 \mathrm{~mL}), 160{ }^{\circ} \mathrm{C}, 24 \mathrm{~h}$ under argon unless otherwise noted.

${ }^{b} \mathrm{GC}$ yields based on tributylamine. ${ }^{c}$ Reaction was carried out at $150{ }^{\circ} \mathrm{C} .{ }^{d} 2$ equiv. 1a was used. ${ }^{e} 5 \mathrm{~mol} \%$ catalyst was used.

溶剂对反应的影响(表 1, Entries 13 17), 当选用弱极性 溶剂甲苯时, 能较好地促进反应的进行, 但是在强极性 溶剂 DMF, DMSO 和 NMP 中反应活性不是很高, 尤其 是在 DMSO 中不能进行反应; 在无溶剂的情况下也能
很好地进行反应，产率能达到 72\%(表 1, Entry 17)，但是 考虑到底物拓展方面有部分硝基苯化合物是固体，可能 在反应的溶解性方面受到影响，因此我们选择以弱极性 的甲苯为溶剂. 另外，反应温度、反应物的用量比、催 化剂的用量对反应也有一定的影响(表 1, Entries 18 20).

\section{2 反应底物拓展}

在获得最佳条件之后，我们对底物进行了一系列的 扩展. 首先，我们考察了三正丁胺和带不同取代基的芳 香硝基化合物的反应. 其次, 考察了对硝基甲苯和不同 链长的三级胺(包括二级胺)的反应, 以此来获悉底物结 构对反应产生的影响, 结果如表 2 所示.

由表 2 的数据可知, 当苯环上不带任何取代基时, 反应能很好的进行，产率能达到 68\%(表 2, Entry 2). 当 用对位是甲氧基作为强给电子基时对反应的影响不大, 产率也能达到 54\%(表 2, Entry 3), 遗憾的是当苯环的对 位为卤素原子时产率比较低(表 2, Entries 4,5), 可能的 原因是在反应过程中的低价钓把对位的卤素还原了. 但 是苯环的间位有取代基时并不能影响反应的进行，能得 到较高的产率，特别是间位为碘取代时产率也能达到 $63 \%$, 这为在喹啉上进一步进行修饰提供了很好的途 径. 硝基苯的邻位有取代基时产率很低, 可能是邻位取 代基的位阻太大所导致的. 值得一提是，选用稠环硝基 苯时产率能达到 $82 \%$, 这为合成苯并喹啉一些衍生物提 供一条新的途径. 接着我们也考察了一些杂环的硝基化 合物，其中 6-硝基噻唑能较好的进行反应，产率能达到 $62 \%$. 最后我们还考察不同链长的三级胺对反应的影 响, 发现链长越长时, 反应产率较高(表 2, Entries 12

表 2 喹啉衍生物的合成 ${ }^{a}$

Table 2 Syntheses of quinolines

Entry




\begin{tabular}{|c|c|c|c|c|}
\hline Entry & Nitroarene & Amine & Product & Yield $^{b} \%$ \\
\hline 5 & & $\mathrm{~N}(n-\mathrm{Bu})_{3}$ & $3 \mathrm{e}$ & 32 \\
\hline 6 & 1f & $\mathrm{N}(n-\mathrm{Bu})_{3}$ & ST & 75 \\
\hline 7 & $1 \mathrm{~g}$ & $\mathrm{~N}(n-\mathrm{Bu})_{3}$ & 39 & 63 \\
\hline 8 & & $\mathrm{~N}(n-\mathrm{Bu})_{3}$ & $3 \mathrm{~h}$ & $12^{c}$ \\
\hline 9 & & $\mathrm{~N}(n-\mathrm{Bu})_{3}$ & $3 \mathbf{i}$ & 40 \\
\hline 10 & & $\mathrm{~N}(n-\mathrm{Bu})_{3}$ & 3 & 82 \\
\hline 11 & 1k & $\mathrm{N}(n-\mathrm{Bu})_{3}$ & 31 & 62 \\
\hline 12 & $1 \mathrm{a}$ & $\mathrm{NPr}_{3}$ & & 52 \\
\hline 13 & 1a & $\mathrm{N}\left(n-\mathrm{C}_{6} \mathrm{H}_{13}\right)_{3}$ & $3 m$ & 74 \\
\hline 14 & $1 \mathrm{a}$ & $\mathrm{N}\left(n-\mathrm{C}_{8} \mathrm{H}_{17}\right)_{3}$ & $3 n$ & 75 \\
\hline 15 & 1a & $\mathrm{NH}(n-\mathrm{Bu})_{2}$ & & 35 \\
\hline
\end{tabular}

${ }^{a}$ Conditions: nitroarene $(0.6 \mathrm{mmol})$, amine $(0.2 \mathrm{mmol}), \mathrm{RuCl}_{3} \bullet 3 \mathrm{H}_{2} \mathrm{O}(0.02 \mathrm{mmol}), \mathrm{PCy}_{3} \mathrm{HBF}_{4}(0.04 \mathrm{mmol})$, toluene $(0.2 \mathrm{~mL}), 160{ }^{\circ} \mathrm{C}, 24 \mathrm{~h}$ under argon. ${ }^{b}$ Isolated yields based on amines. ${ }^{c} \mathrm{GC}$ yields based on tributylamine.

14). 但用二级胺反应反应时产率明显下降(表 2, Entry

15), 可能是二级胺提供的氢不够.

\section{3 反应机理的探讨}

实验中通过 GC-MS 发现, 反应结束后不仅得到了 喹啉衍生物, 还得到了少量的亚胺(图 1)以及微量的苯 胺. 结合前期的研究工作, 我们推断可能的反应机理如
图 1 所示, 首先三氯化钉在配体的作用下活化生成低价 的钉, 然后三正丁基胺(2a)在低价钉的催化下脱氢生成 中间体 4, 同时脱下来的氢将硝基苯部分还原, 通过循 环 $\mathrm{A}$ 路线中间体 $\mathbf{4}$ 与亲核性强的苯胺加成生成中间体 5, 在钓的催化作用下 $\mathrm{C}-\mathrm{N}$ 断裂生成 $N$-亚丁基苯胺钉 配合物 6. $N$-丁基苯胺 ${ }^{[19]}$ 与低价钉配位物 6 络合生成中 


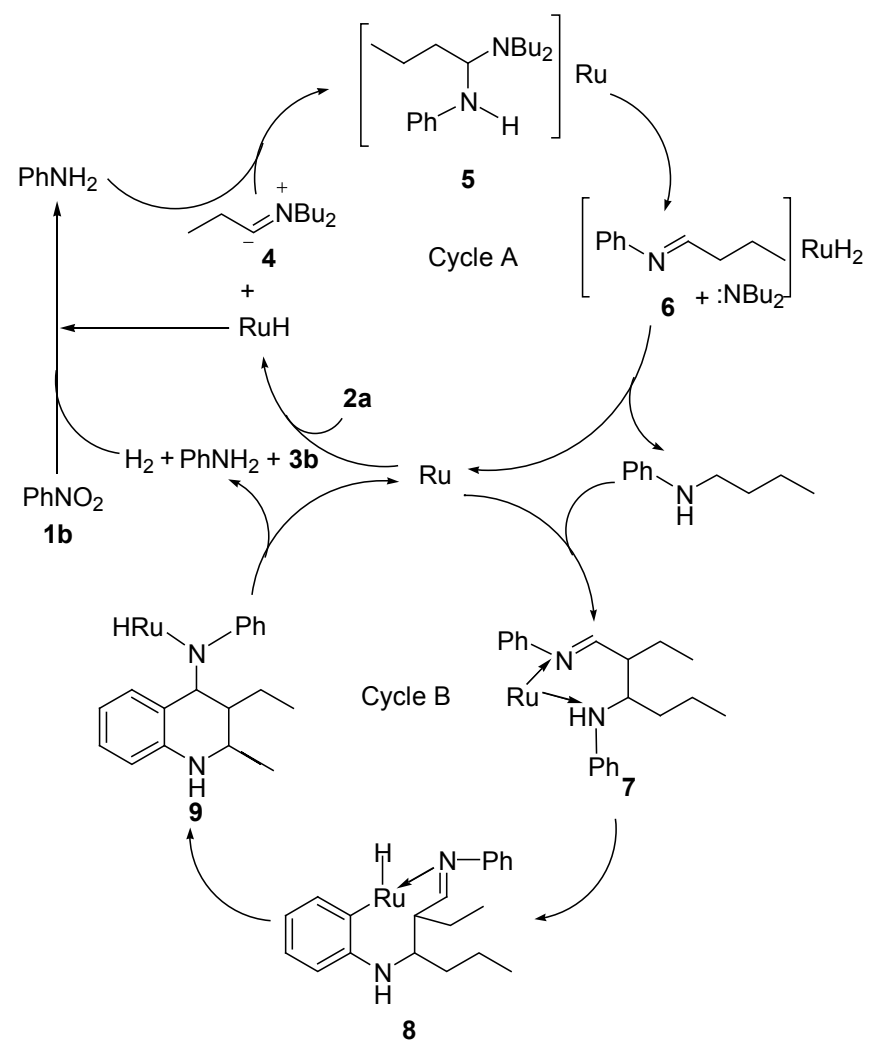

图 1 一锅法合成喹啉衍生物的可能反应机理

Figure 1 Proposed mechanism for one-pot synthesis of quinolines

间体 7, 接着通过循环 $\mathrm{B}$ 路线在金属钉的催化下进行一 系列的成环脱氢形成中间体 $\mathbf{8}^{[20]}$ 和 $\mathbf{9}^{[21]}$, 最后在钉的作 用下苯胺离去, 生成产物 $3 \mathrm{~b}$, 同时释放出来的氢进一步 还原硝基苯，而释放的低价钓参与循环 $\mathrm{A}$ 路线.

\section{3 结论}

本文发现了以芳香硝基化合物和脂肪三级胺为底 物, 三环己基膦四氟硼酸盐为配体, 在三氯化钉的催化 下一锅合成喹啉衍生物的方法. 该法不需添加任何额外 的还原剂, 原料便宜易得, 操作工艺简单, 产率中等.

\section{References}

[1] (a) Wei, X. L.; Su, R. B. Bull. Acad. Millitry Med. Sci. 2002, 26, 228 (in Chinese).

(魏晓莉, 苏瑞斌, 军事医学科学院院刊, 2002, 26, 228.)

(b) Mikhailitsyn, F. S.; Kozyreva, N. P.; Rabinovich, S. A.; Maksakovskaya, Y. V.; Kulikovskaya, I. M.; Dadasheva, N. R.; Lebedeva, M. N.; Bekhli, A. F.; Lychko, N. D.; Uvarova, N. A. Med. Parazitol. Parazit. Bolezni 1992, 1, 50.

[2] Via, L. D.; Gia, O.; Gasparotto, V.; Ferlin, M. G. Eur. J. Med. Chem. 2008, 43, 429.

[3] Prescott, T. A. K.; Sadler, I. H.; Kiapranis, R. J. Ethnopharmacol. 2007, 109, 289.

[4] Iida, H.; Yuasa, Y.; Kibayashi, C. J. Org. Chem. 1979, 44, 3985.

[5] (a) Wu, Y. C.; Liu, L.; Li, H. J.; Wang, D.; Chen,Y. J. J. Org. Chem. 2006, 71, 6592 (b) Denmark, S. E.; Venkatraman, S. J. Org. Chem. 2006, 71, 1668

[6] Jutz, C.; Wagner, R. M. Angew. Chem., Int. Ed. Engl. 1972, 11 , 315.

[7] (a) Kaslow, C. E.; Summers, R. M. J. Org. Chem. 1955, 20, 1738. (b) Hause, C. R.; Murray, J. G. J. Am. Chem. Soc. 1955, 77, 2851.

[8] (a) Yang, D. Q.; Lü, F.; Guo, W. Chin. J. Org. Chem. 2004, 24, 366 (in Chinese).

(杨定乔, 吕芬, 郭维, 有机化学, 2004, 24, 366.)

(b) Wu, L. H.; Yang, D. Q. Chin. J. Org. Chem. 2010, 30, 1180 (in Chinese).

(吴利欢，杨定乔，有机化学，2010, 30, 1180.)

[9] Shindoh, N.; Tokuyama, H.; Takemoto,Y.; Takasu, K. J. Org. Chem. 2008, 73, 7451.

[10] (a) Zhang, W. W.; Wang, X. S.; Li, Q.; Yao, C. S.; Tu, S. J. Chin. J. Org. Chem. 2008, 28, 881 (in Chinese).

(张梅梅, 王香善, 李庆, 姚昌盛, 屠树江, 有机化学, 2008, 28, 881.)

(b) Mali, J. R.; Pratap, U. R.; Jawale, D. V.; Mane, R. A. Tetrahedron Lett. 2010, 51, 3980.

[11] (a) Wang, H. S.; Zeng, J. E. Chin. J. Org. Chem. 2010, 30, 1072 (in Chinese).

(王宏社，曾君娥，有机化学，2010, 30, 1072.)

(b) Hollmann, D.; Bahn, S.; Tillack, A.; Beller, M. Chem. Commun. 2008, 44, 3199 .

(c) Lubinu, M.; Luca, L.; Giacomelli, G.; Porcheddu, A. Chem. Eur. J. 2011, 17, 82 .

(d) Lang, X. J.; Ji, H. W.; Chen, C. C.; Ma, W. H.; Zhao, J. C. Angew. Chem., Int. Ed. 2011, 50, 3934.

(e) Liu, L. H.; Zhang, S. Y.; Fu, X. F.; Yan, C. H. Chem. Commun. 2011, 47, 10148.

(f) Saidi, O.; Blacker A.; Farah, M.; Marsdon, S.; Williams, J. 
Angew. Chem., Int. Ed. Engl. 2009, 48, 7375.

[12] (a) Feng, C.; Liu, Y.; Peng, S. M.; Shuai, Q.; Deng, G. J.; Li, C. J. Org. Lett. 2010, 12, 4888.

(b) Liu, Y.; Chen, W.; Feng, C.; Deng, G. J. Chem. Asian J. 2011, $6,1142$.

(c) Xiao, F. H.; Liu, Y.; Tang, C. L.; Deng, G. J. Org. Lett. 2012, 14, 984 .

[13] Watanabe, Y.; Shim, S. C.; Mitsudo, T. Bull. Chem. Soc. Jpn. 1981, 54,3460 .

[14] Nakajima, T.; Inada, T.; Igarashi, T.; Sekioka, T.; Shimizu, I. Bull. Chem. Soc. Jpn. 2006, 79, 1941.

[15] Case, J. J. Chem. Eng. Data 1968, 13, 568.

[16] Yamamoto, I.; Furukawa, T.; Nakajima, H.; Gotoh, H. J. Chem.
Soc., Perkin Trans. 1 1976, 1597.

[17] Wang, D.; Wang, X.; Wang, D.; Lu, S.; Zhou, Y.; Li, Y. J. Org. Chem. 2009, 74, 2780.

[18] Cho, C.; Lee, N.; Kim, T.; Shim, S. J. Heterocycl. Chem. 2004, 41, 423.

[19] (a) Basu, A.; Bhaduri, S.; Sharma, K.; Jones, P. G. J. Chem. Soc., Chem. Commun. 1987, 1126.

(b) Wang, G. Z.; Bäckvall, J. E. J. Chem. Soc., Chem. Commun. 1992, 980 .

[20] Kharasch M. S.; Richlin, I.; Mayo, F. R. J. Am. Chem. Soc. 1940, $62,494$.

[21] Watanabe, Y.; Tsuji, Y.; Ohsugi, Y.; Shida, J. Bull. Chem. Soc. Jpn. 1983, 56, 2452.

(Lu, Y.) 